\title{
Approximate Methods for Solving Differential Equations for Continuous Models of a Sustainable Economy
}

\author{
Tatyana Gorskaya ${ }^{1, *}$, Anis Galimyanov ${ }^{2}$ \\ ${ }^{1}$ Kazan State University of Architecture and Engineering, Kazan, Russia \\ ${ }^{2}$ Kazan (Volga) Federal University, Kazan, Russia \\ *Corresponding author. Email: gorskaya0304@mail.ru
}

\begin{abstract}
The article provides an overview of the economic situations of different countries related to the impact of the COVID19 pandemic and reveals some conclusions for the economies of the countries. An overview of some economic models based on the application of ordinary differential equations, linear and nonlinear, integer and fractional orders is presented. An overview of approximate methods for their solution is also presented. The expediency of application of this or that numerical method for solution of differential equations is shown. For some equations of continuous models of economy an approximate method is proposed and substantiated in the paper. The convergence estimations of the approximate methods in the corresponding functional spaces are given. The implementation of the proposed solution methods on test examples are presented. Also the results of numerical solution of these equations have been obtained in the form of graphs and a comparative analysis of the obtained results has been carried out.
\end{abstract}

Keywords: continuous models of economics, differential equations, approximate methods.

\section{INTRODUCTION}

The economy reacts particularly sharply to global world problems that affect practically every country in the world. Such a global challenge to humanity was the outbreak of the COVID-19 pandemic, which caused great economic losses in many countries, undermining some external economic contacts of countries, caused damage to human resources, claiming millions of lives, revealed problems in the social spheres. Despite its destructive nature, the pandemic stimulated the development of domestic economies, so, for example, the U.S. [1] saw the shortest recession, which passed faster than during the global GFC crisis, thanks to the economic stimulus programs. In addition, the problems of the economy have revealed, exposed its most vulnerable sides, the agendas of the Security Councils of many countries, including Russia, is the issue of improving living standards by developing social programs and encouraging small businesses, because entrepreneurship plays a key role in the economic development of countries. Statistics show [2] that in times critical for the economy, the opportunities for entrepreneurship are limited and for their intensification requires the introduction of innovative approaches. Thus, the crisis associated with COVID-19 was a socalled catalyst for the development of new businesses. As noted earlier, there was a decline in economic performance due to difficulties in international communications. In connection with this, we should also note the migration policy. The labor markets of a number of countries were deprived of workers, and they, in turn, were deprived of income. In Tajikistan, for example, before the pandemic, external cash inflows exceeded a quarter of the country's annual GDP. But as studies have shown [3], the pandemic had only a temporary impact, serious only after the outbreak, and then the process of migration and remittances recovered, which determined its persistent nature. An important aspect of creating a sustainable economy is to identify models that are resilient to adverse situations. In this connection, it is possible to cite a number of works $[4,5]$, the authors of which conducted research in this area, defined scenarios for the development of territorial economic systems [6]. The crisis aspects of globalization in the development of financial relations 
require, in the opinion of the authors [7], the development of methods that allow forecasting the dynamics of economic cycles, the model of which uses systems of differential equations. Also the model based on the system of differential equations is used by the authors [8] on the basis of statistical data of the US GDP defined a predictive model linking several economic indicators.

Continuous models of the economy are mainly based on integral order differential equations. For example, in [9] a model of stocks and its numerical solution is presented, there to the traditional model is connected a component such as memory effect. Dynamic processes in the economy are also investigated in [10], and a new differential equation describing the dynamics is added to the basic system of differential equations, the model is illustrated by an example and a numerical solution is found. In addition, there are many models that use a probabilistic component, stochastic models. For example, for the model of optimal investment application, in [11] a numerical solution and analysis of the results. In [12] the authors studied demand forecasting, a model based on stochastic differential equations. It may also be noted that in addition to continuous models of the economy based on linear differential equations and their systems, there are also two-dimensional models, such as, [13, 14], which involve partial differential equations, but we will focus only on ordinary linear differential equations, the range of coverage of economic models which are diverse. For processes accompanied by jumps, differential equations of fractional order of differentiation are used, economic problems with jumps refer to the problems of sustainable economy. Economic models such as the Solow model were considered by the authors [15] to describe the trajectory of economic growth. The Haavelmo model of economic growth, a nonlinear macroeconomic model with nonlinear fractional-order differentials, was considered by the authors [16].

The solution of differential equations in some models can be obtained precisely, somewhere it is possible to find an approximate solution, the accuracy of which can also be established. In addition, the accuracy of the information underlying the model also determines the accuracy of the results obtained. Thus, in [17] the authors on the fact that the study of the processes of the real economy does not take into account the existence of the shadow economy. In [18] it was proposed to use the principle of detection and elimination of redundant elements in the mathematical model. The main approximate apparatus for obtaining a numerical solution of a differential equation is the Runge-Kutta method, which is actively used. In [19], the authors carry out a comparative analysis of the results obtained using the Euler, Runge-Kutta 2, and Runge-Kutta 4 methods. Runge-Kutta showed good calculation accuracy. At the same time, it is necessary to note the theoretical aspect of the research. While the RungeKutta method used, for example, in [20], despite its labor-intensive nature, has considerable accuracy and is widely used in computer calculations, it cannot be applied to theoretically substantiate an approximate solution for a wide class of problems. Where as direct and projective methods have this quality. Of the large variety of direct methods [21] we can single out a group of projection methods, which includes the Galerkin method, which is convenient for theoretical justification of existence and estimation of approximate solution error, however, it is difficult to implement in practice. The application of direct and projective methods for solving equations (Ritz, Bubnov-Galerkin methods) has been recently devoted to many works [22]. This is due to the fact that the issues of theoretical research of these equations and the possibility of finding the optimal solution are of great interest for modern science. Also, despite the results obtained for the study of differential equations with fractional order [23], there are currently very acute problems of finding and justifying approximate methods for their solution.

\section{MATERIALS AND METHODS}

Consider the application of differential equations to continuous models of the economy, for example, when studying the dynamics of capital formation (in the neoclassical growth model), it is represented as a function of time $t$ and the following nonlinear first-order differential equation with separating variables is obtained:

$$
k^{\prime}=l f(k)-(\alpha+\beta) k .
$$

Labor productivity as a function of time is defined through $\mathrm{Y}=\mathrm{F}(\mathrm{K}, \mathrm{L})$ - national income, where $\mathrm{F}$ is a homogeneous production function of the first order, for which the following relation is true: $\mathrm{F}(\mathrm{TK}, \mathrm{TL})=\mathrm{TF}(\mathrm{K}$, $\mathrm{L}$ ), $\mathrm{K}$ is the amount of capital investments (production funds), $\mathrm{L}$ is the amount of labor input. Let us consider the value of capital investment $\mathrm{K}=\mathrm{K} / \mathrm{L}$, then labor productivity is expressed by the formula

$$
f(k)=\frac{F(K, L)}{L} .
$$

Let's consider a specific problem: for the production function $F(K, L)=\sqrt{K L}$. find the integral curves of 
equation (1) and the stationary solution. Please refer to "Equation (2), from which it follows that

$$
F(k)=\sqrt{k},
$$

and then Equation (1) has the form

$$
\frac{d k}{d t}=l \sqrt{k}-(\alpha+\beta) k
$$

Consider the problem for a differential equation of the form:

$$
\begin{gathered}
-\frac{d^{2} u}{d x^{2}}+\frac{d u}{d x}+q u=f(x), \quad x \in(0,1), \quad q>0, \\
u(0)=u(1)=0,
\end{gathered}
$$

or in the operator form: $A u+B u=f$, where $A u=-d^{2} u / d x^{2}, B u=d u / d x+q u$. As D(A), let us take the set of twice continuously differentiable functions on $(0,1)$ that satisfy the given boundary conditions. The energy space generated by the operator $A$ : $H_{A}$ - space of function in $W_{2}^{1}$, satisfying the given boundary conditions. As the basis functions we can take a system of functions $\varphi_{i}=\operatorname{sini\pi x} x$, full in space $H_{A}$.

Then an approximate solution of equation (4), according to [24], is found as:

$$
u_{N}=\sum_{i=1}^{N} a_{i} \sin i \pi x .
$$

The unknown coefficients $a_{i}$ of formula (5) are determined according to the Bubnov-Galerkin method from a system of linear algebraic equations written in matrix form: $L a=f$, где $L=\left\{L_{i j}\right\}, \quad f=\left(f_{1}, \ldots, f_{N}\right)^{\mathrm{T}}$, where

$$
\begin{gathered}
L_{i j}=\int_{0}^{1}\left(\frac{d \varphi_{i}}{d x} \frac{d \varphi_{j}}{d x}+\frac{d \varphi_{j}}{d x} \varphi_{i}+q \varphi_{i} \varphi_{j}\right) d x, \\
f_{i}=\left(f, \varphi_{i}\right)=\int_{0}^{1} f(x) \varphi_{i}(x) d x .
\end{gathered}
$$

Then the following theorem is valid for equation (4).

Theorem. Let the operator $A^{-1} B$ is quite continuous in the $H_{A}$. Then at sufficiently large $\mathrm{N}$ the BubnovGalerkin method gives the only approximate solution $u_{N}$, which converges to the generalized solution $u$ of equation (4) on the norm of the space $H_{A}$.

The proof of the theorem is carried out according to [24].

\section{RESULTS AND DISCUSSION}

As an illustration, let us give examples of solutions of some differential equations for models of economics using two approximate methods, namely the RungeKutta method and the Bubnov-Galerkin method. As the first example, let us give the solution of a nonlinear differential equation (3) by the Runge-Kutta method. Let us take in equation (3) $\alpha=2 ; \beta=1,5 ; \mathrm{L}=10$ and in the initial moment $\mathrm{Y}=2$, then we find an approximate solution as shown on Figure 1:

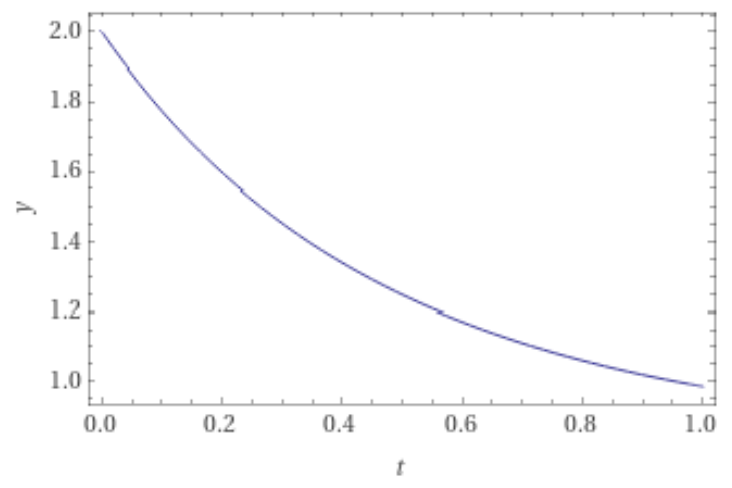

Figure 1 Graph of the integral curve-solution of equation (3) by the Runge-Kutta method.

The exact solution of equation (3) with the above parameters is found analytically, by solving the Cauchy problem for the equation with separating variables $49 y-28 \sqrt{10 y}=(138-56 \sqrt{5}) e^{-3.5 x}-40$, the graph is shown in figure 2 .

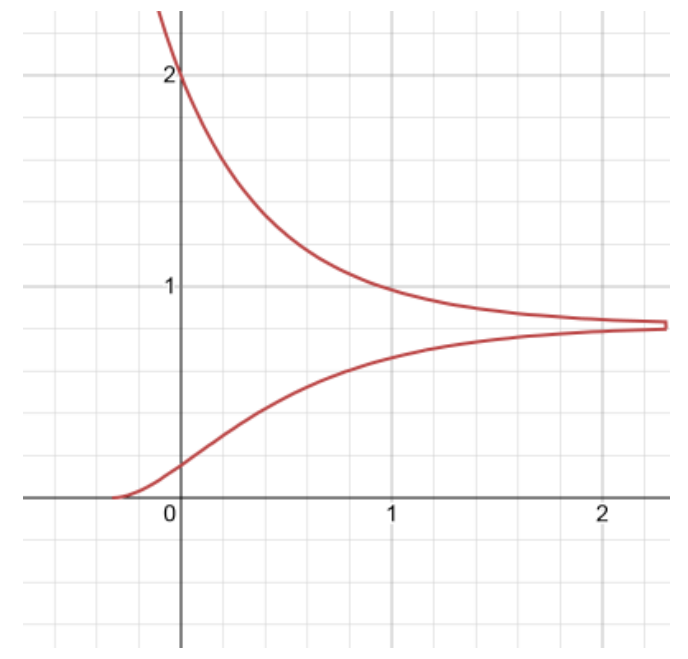

Figure 2 The graph of the exact solution of the equation (3).

Note that the graph in Figure 2 shows two branches of this function, since the exact solution is represented as a quadratic function with respect to the new desired $\sqrt{y}$, which has two branches of the graph, due to symmetry with respect to the straight line $y=\frac{40}{49^{\prime}}$ however, the upper part of the graph coincides completely with the approximate solution in Figure 1. 
Thus, as shown above in the example of the solution of equation (3), with the input parameters of the problem $\mathrm{L}, \alpha$ and $\beta$ unchanged, the function of capital equipment in this case steadily tends to the stationary value regardless of the initial conditions. This agrees with the known results from economic theory [25].

As an illustration of the following approximation methods, consider a linear differential equation for a market model with predicted prices.

$P^{\prime \prime}+2 P^{\prime}+5 P=15$.

Consider the case when at the initial moment $\mathrm{P}(0)=4$ and $\mathrm{P}^{\prime}(0)=1$, then find the exact solution of the linear inhomogeneous differential equation of the second order with respect to the function $\mathrm{P}(\mathrm{T})$. It is known that the exact solution of the Cauchy problem is: $P(T)=e^{-T}(\sin (2 T)+\cos (2 T))+3$, the solution graph in Figure 3.

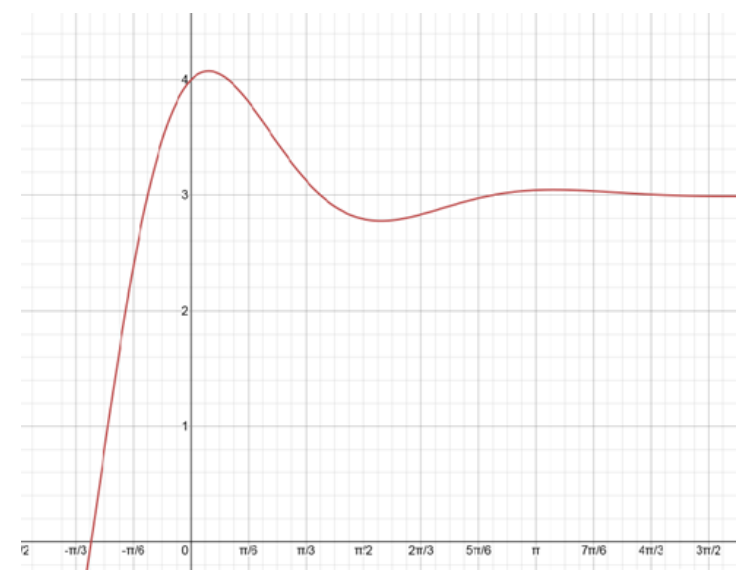

Figure 3 The graph of the exact solution of the equation (6).

Analyzing the obtained result, it was found that all prices tend to the established price $\mathrm{PSt}=3$ with fluctuations around it, and the amplitude of these fluctuations decays with time.

An approximated solution found by the Runge-Kutta method of the fourth order of accuracy has the form shown in Figure 4.

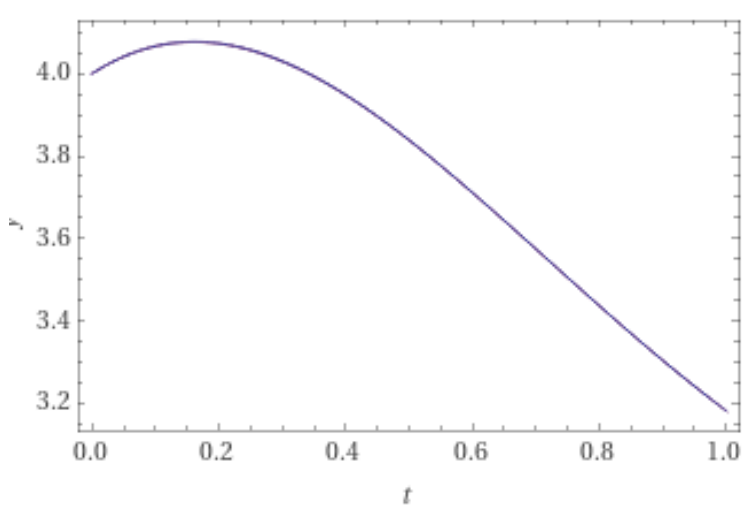

Figure 4 Graph of the approximate solution of equation (6) by the Runge-Kutta method.

Comparing the graphs of the exact and approximate solutions, we can see that they coincide, which shows that the accuracy of the approximate solution is high.

However, in the general case for theoretical justification of the application of approximate methods of solution of differential equations, additional research is needed.

Next, we calculate an approximate solution using the computational scheme of the Bubnov-Galerkin method proposed for equation (4), according to which the solution is reduced to a system of linear algebraic equations $A u=F$. Elements of matrix A, the vectorcolumn of free members $\mathrm{F}$, the elements of which are in the form $F_{i}=\int_{0}^{1} 15 \sin (i \pi x) d x$, calculated in Mathcad.

As a result, an approximate solution $\mathrm{U}(\mathrm{x})$ according to formula (5) is obtained at $\mathrm{N}=10$, the graph of which is shown in Figure 5.

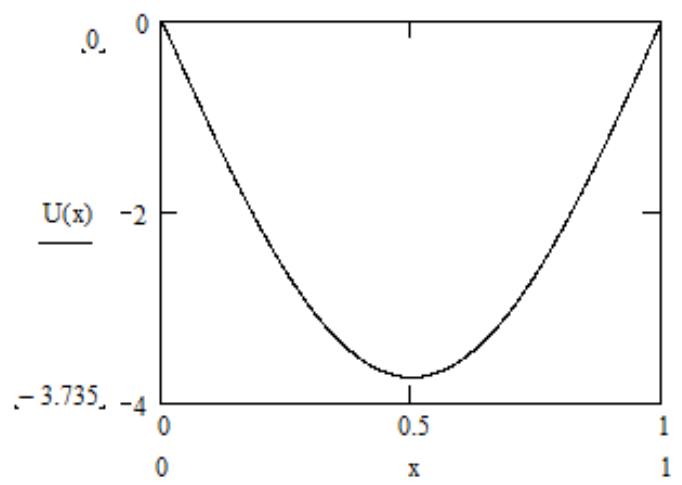

Figure 5 Graph of the approximate solution of equation (4) by the Bubny-Galerkin method.

Let us compare the approximate solution of equation (4) with the exact solution of the equation at given initial conditions. We have the exact solution in the form: 
$\mathrm{u} 1(\mathrm{x}):=-3 \cdot \mathrm{e}^{-\mathrm{x}} \cdot \cos (2 \cdot \mathrm{x})+\left(3 \cdot \frac{1}{\tan (2)}-\frac{3 \cdot \mathrm{e}}{\sin (2)}\right) \cdot \mathrm{e}^{-\mathrm{x}} \cdot \sin (2 \cdot \mathrm{x})+3$

The graph of the exact function is shown in Figure 6 and agrees completely with the graph of the approximate solution in Figure 5, obtained by the Bubnov-Galerkin method.

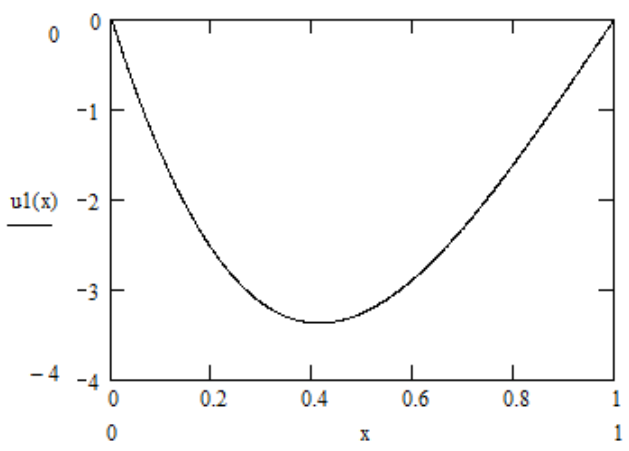

Figure 6 The graph of the exact solution of equation (6).

Consider the task of:

$D^{(\alpha)} u+q u=f(x), \quad x \in(0.1)$,

$u(0)=u(1)=0$.

he approximate solution of (7) will be searched for by the Ritz method [24], according to which we take the eigen functions of the operator as basis functions $A=D^{(\alpha)}+q$ with the area of definition $D(A)$, consisting of continuous on $[0,1]$ and having derivatives up to and including the second order of functions $u(x)$, satisfying the conditions $u(0)=u(1)=0$. Note that the eigenvalue $\lambda_{i}$ and the corresponding eigen function $\varphi_{i}$ of operator A have the form $\lambda_{i}=i^{\alpha} \pi^{\alpha}+q, \varphi_{i}=\sin i \pi x, i=1,2, \ldots$. Then an approximate solution of (7) will be sought in the form (5), where the unknown coefficients of expansion (5) are defined explicitly as:

$$
\alpha_{i}=\frac{2}{i^{\alpha} \pi^{\alpha}+q} \int_{0}^{1} f(x) \sin i \pi x d x .
$$

Let us take as a test example the numerical implementation of the proposed computational scheme for problem (7) values $\alpha=1,5, q=1$.

Then equation (7) will take the form:

$$
\begin{aligned}
& D^{(1.5)} u+u=f(x), \\
& x \in(0.1), \quad u(0)=u(1)=0
\end{aligned}
$$

where the right-hand side of equation (9) is explicitly specified as a function $f(x)=\left(1+b^{1.5}\right) \sin b x-\left(1+(\pi-b)^{1.5}\right) \sin (\pi-b) x$.

Obviously, the exact solution is $u(x)=\sin b x-\sin (\pi-b) x$.
As an illustration of the method, the approximate solutions of (7) will be found in the form of the trigonometric polynomial (5), the unknown coefficients of which will be found from the definite integral (8) and after slight transformations will finally take the form:

$\alpha_{i}=\frac{2 \pi i \cdot \sin b \cdot(-1)^{1}}{i^{1,5} \pi^{1,5}+1} \cdot\left[\frac{\left(1+b^{1.5}\right)}{b^{2}-i^{2} \pi^{2}}-\frac{\left(1+(\pi-b)^{1.5}\right)}{(\pi-b)^{2}-i^{2} \pi^{2}}\right]$.

Substituting the formula we obtain an approximate solution for which the graph is shown in Figure 7. The graph of the corresponding exact solution is shown in Figure 8 .

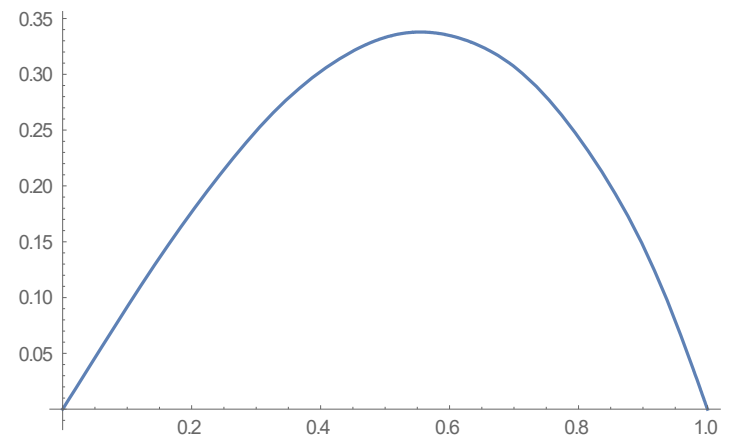

Figure 7. A graph of the exact solution $u(x)$.

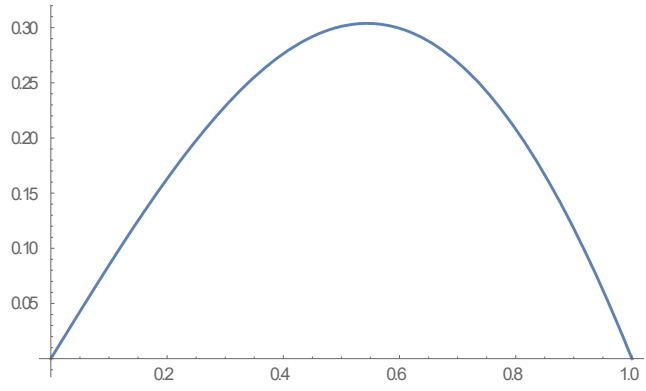

Figure 8. Graph of the approximate solution $u_{10}(x)$.

It is easy to see that the graphs of the approximate and exact solutions almost coincide.

\section{CONCLUSIONS}

When investigating various differential equations for continuous models of the economy, it is necessary to understand that the Runge-Kutta method of the fourth order is the most used one for ordinary differential equations. The Bubnov-Galerkin method, which refers to direct methods that in turn lead to systems of linear equations, is also now widely used to justify the method, to obtain theoretical estimates of the accuracy of the method, and to subsequently solve the equations. However, for some equations, one method gives a smaller error and for others a different one. However, there are a number of problems, such as problems with differential equations of fractional order, which cannot be solved by the Runge-Kutta method, so for them the 
authors justified the application of the Bubnov-Galerkin method.

\section{REFERENCES}

[1] A. N. Berger, A. Demirgüç-Kunt, Banking research in the time of COVID-19, in: Journal of Financial Stability, 57, 100939 https://doi.org/10.1016/j.jfs.2021.100939.

[2] Z. Goschin, M. Antonia, H. Tigau, Entrepreneurship recovery in Romania after the great recession, in: A dynamic spatial panel approach Sustainability, 13(19), 10702 (2021), https://doi.org/10.3390/su131910702.

[3] S. Shimizutani, E. Yamada, Resilience against the pandemic: The impact of COVID-19 on migration and household welfare, in: Tajikistan PLoS ONE 16, $\quad \mathrm{e} 0257469 \quad$ (2021). https://doi.org/10.1371/journal.pone.0257469.

[4] A. Mendoza-Velázquez, L. Rendón-Rojas, Identifying resilient industries in Mexico's automotive cluster: Policy lessons from the great recession to surmount the crisis caused by COVID 19, in: Growth and Change, 52(3), pp. 1552-1575, (2021). https://doi.org/10.1111/grow.12515.

[5] S. Quatrini, Challenges and opportunities to scale up sustainable finance after the COVID-19 crisis, in: Lessons and promising innovations from science and practice Ecosystem Services 48, 101240

(2021). https://doi.org/10.1016/j.ecoser.2020.101240.

[6] M. E. Morales, S. Lhuillery, Modelling circularity in bio-based economy through territorial system dynamics 2021, in: IEEE European Technology and Engineering Management Summit, E-TEMS 2021 - Conference Proceedings, pp. 161-165 (2021). https://doi.org/10.3390/su132111636.

[7] A. P. Gorbunov, T. V. Kasaeva, A. P. Kolyadin, L. D. Tokova, Forecasting the Economic State of a Bank on the Basis of Bankruptcy Indicator, in: Advances in Intelligent Systems and Computing, 726, pp. 639-645 (2019). https://doi.org/10.1007/978-3-319-90835-9_74.

[8] D. Rybski, P. Pradhan, S. T. Shutters, V. Butsic, J. P. Kropp, Characterizing the sectoral development of cities, in PLoS ONE 16, e0254601 (2021). https://doi.org/10.1371/journal.pone.0254601.

[9] R. Pakhira, U. Ghosh, S. Sarkar, V. N., Mishra, Study of Memory Effect in an Economic Order
Quantity Model for Completely Backlogged Demand During Shortage, in: Progress in Fractional Differentiation and Applications, 7(3), (2021), pp. 177-190, https://doi.org/10.18576/pfda/070305.

[10] E. V. Nikolova, Chaos in a generalized system for modeling dynamics of three interacting economic sectors, in: AIP Conference Proceedings 2321, 030025, (2021). https://doi.org/10.1063/5.0040086.

[11] M. A. Baten, R. Khalid, A Stochastic Control Model of Investment and Consumption with Applications to Financial Economics, in: Stochastics and Quality Control, 35(2), (2020), pp. 43-55. https://doi.org/10.1515/eqc-2020-0017.

[12] J. Sowinski, Forecasting of electricity demand in the region, in: E3S Web of Conferences, 84(4), 01010 .

https://doi.org/10.1051/e3sconf/20198401010.

[13] F. Torres-Bejarano, C. Couder-Castañeda, H. Ram-Rez-León, I.E. Herrera-Díaz，H. BarriosPiña, Numerical modelling of heavy metal dynamics in a river-lagoon system, in: Mathematical Problems in Engineering, 8485031 (2019). https://doi.org/10.1155/2019/8485031.

[14] V. Shaydurov, V., Kornienko, S. Zhang, The Euler-Lagrange Approximation of the Mean Field Game for the Planning Problem, in: Lobachevskii Journal of Mathematics, 41(12), (2020), pp. 27022713, https://doi.org/10.1134/S1995080220120380.

[15] Z. Lin, H. Wang, Modeling and application of fractional-order economic growth model with time delay, in: Fractal and Fractional, 5(3), (2021), p. 74, https://doi.org/10.3390/gractalfract5030074.

[16] V. E. Tarasov, Nonlinear growth model with long memory: generalization of Haavelmo model, in: Nonlinear Dynamics, 104(4), (2021), pp. 44134425, https://doi.org/10.1007/s11071-021-06484-2.

[17] A. Musayev, A. Musayeva, A Study of the Impact of Underground Economy on Integral Tax Burden in the Proportional Growth Model under Uncertainty, in: Advances in Fuzzy Systems, 6309787

(2018). https://doi.org/10.1155/2018/6309787.

[18] Y. Matviychuk, O. Matviychuk, Reduction principle of the mathematical model and its applications, in: 2018 14th International Conference on Perspective Technologies and Methods in MEMS Design, MEMSTECH 2018 - 
Proceedings, pp. 174-177 (2018). https://doi.org/10.1109/MEMSTECH.2018.836572 7.

[19] M. Rahaman, S. P., Mondal, A. A., Shaikh, N. Senu, S. Salahshour, Arbitrary-order economic production quantity model with and without deterioration: generalized point of view, in: Advances in Difference Equations, 1, 16 (2020). https://doi.org/10.1186/s13662-019-2465-x.

[20] V. L. Vorontsova, A. G. Bagoutdinova, A. F. Galemzianov, Numerical modeling of hydrodynamic processes in screw tubes, in: Journal of Advanced Research in Dynamical and Control Systems, 11(5), (2019), pp. 38-43.

[21] I. V. Semushin, Numerical methods of algebra (2006).

[22] A. F. Galimyanov, V. L. Vorontsova, T. Y. Gorskaya, Approximate methods for the equations with fractional differential operator, in: Global Journal of Pure and Applied Mathematics, 11(6), (2015), pp. 5133-5144.

[23] A. Galimyanov, T. Gorskaya, Fractional Order Integrals for the Sustainable Development Model, in IOP: Conference Series: Materials Science and Engineering, $890(1), \quad 012180 \quad$ (2020). https://doi.org/10.1088/1757-899X/890/1/012180.

[24] G. I. Marchuk, V. I. Agoshkov, Introduction to projection-grid methods, p. 416 (1981).

[25] E. G. Zhilyakov, Y. M. Perlov, E. P. Revtova, Fundamentals of econometric data analysis: Textbook (2004). 